\title{
On Determining the Color of the Illuminant using the Dichromatic Reflection Model
}

\author{
Marc Ebner and Christian Herrmann \\ Universität Würzburg, Lehrstuhl für Informatik II, \\ Am Hubland, 97074 Würzburg, Germany, \\ ebner@informatik. uni-wuerzburg.de, \\ http://www2. informatik. uni-wuerzburg. de/staff/ebner/welcome.html
}

\begin{abstract}
The human visual system is able to accurately determine the color of objects irrespective of the spectral power distribution used to illuminate the scene. This ability to compute color constant descriptors is called color constancy. Many different algorithms have been proposed to solve the problem of color constancy. Usually, some assumptions have to be made in order to solve this problem. Algorithms based on the dichromatic reflection model assume that the light reflected from an object results from a combined matte and specular reflection. This assumption is used to estimate the color of the illuminant. Once the color of the illuminant is known, one can compute a color corrected image as it would appear under a canonical, i.e. white illuminant. A number of different methods can be used to estimate the illuminant from the dichromatic reflection model. We evaluate several different methods on a standard set of images. Our results indicate that the median operator is particularly useful in estimating the color of the illuminant. We also found that it is not advantageous to assume that the illuminant can be approximated by the curve of the black-body radiator.
\end{abstract}

\section{Motivation}

A white wall illuminated by yellowish light reflects more light in the red and green part than in the blue part of the spectrum. If we use a camera to take an image of the wall, the sensor of the camera will measure the light reflected from the wall. Thus, a photograph of the wall will have a yellow cast. A human observer, however, is able to somehow discount the illuminant. He will perceive the wall as being white irrespective of the type of illuminant used. This ability to compute color constant descriptors is known as color constancy [1]. Developing algorithms for color constancy is obviously very important for consumer photography. Another area where color constancy algorithms may be used is machine based object recognition. In this paper, we will be looking at several different methods on how to estimate the color of the illuminant from a color image. Once the illuminant is known, it can be used to compute a color corrected image under a canonical, i.e. white illuminant. The different methods will be evaluated on a standard set of test images. 


\section{The Dichromatic Reflection Model}

The dichromatic reflection model assumes that object color is a result of a matte reflection in combination with a specular reflective component $[2,3,4]$. The overall color of the object is determined by the matte reflection whereas specular highlights are caused by the specular reflection. These highlights occur whenever the light is reflected such that it directly enters the camera. Since the light from the light source is reflected directly into the camera it can be used to estimate the color of the illuminant.

Let $\mathbf{S}(\lambda)$ be the vector with the response functions of the sensor. For an RGB-sensor, we have $\mathbf{S}=\left[S_{r}(\lambda), S_{g}(\lambda), S_{b}(\lambda)\right]$ where the functions $S_{i}(\lambda)$ with $i \in\{r, g, b\}$ specify the sensor's response characteristics to light in the red, green, and blue part of the spectrum. Let $E(\lambda)$ be the light falling into the sensor, then the response of the sensor is given by

$$
\mathbf{I}=\int_{-\infty}^{+\infty} E(\lambda) \mathbf{S}(\lambda) d \lambda
$$

Under the dichromatic reflection model, the response of the sensor is given by

$$
\mathbf{I}=\int_{-\infty}^{+\infty}\left(s_{M} R_{M}(\lambda) E(\lambda)+s_{S} R_{S}(\lambda) E(\lambda)\right) \mathbf{S}(\lambda) d \lambda
$$

where $R_{M}(\lambda)$ is the object reflectance with regard to the matte reflection, $R_{S}(\lambda)$ is the object reflectance with regard to the specular reflection, $s_{M}$ and $s_{S}$ are two scaling factors which depend on the object geometry and $E(\lambda)$ is the irradiance falling onto the object [4].

Let us now assume that the response functions are very narrow, i.e. they can be modeled by delta functions $S_{i}(\lambda)=\delta\left(\lambda-\lambda_{i}\right)$. Such ideal sensors only respond to a single wavelength $\lambda_{i}$ with $i \in\{r, g, b\}$. This gives us

$$
I_{i}=s_{M} R_{M, i} E_{i}+s_{S} R_{S, i} E_{i} .
$$

Assuming that the specular reflection behaves like a perfect mirror, i.e. $R_{S, i}=1$, we obtain

$$
I_{i}=s_{M} R_{M, i} E_{i}+s_{S} E_{i} .
$$

Let $\mathbf{C}_{M}=\left[R_{M, r} E_{r}, R_{M, g} E_{g}, R_{M, b} E_{b}\right]$ be the measured matte color of the object and let $\mathbf{C}_{S}=\left[E_{r}, E_{g}, E_{b}\right]$ be the color of the illuminant. We now see that the color measured by the sensor is restricted to the linear combination of the matte color of the object point $\mathbf{C}_{M}$ as seen under illuminant $E$ and the color of the illuminant $\mathbf{C}_{S}$. The two vectors $\mathbf{C}_{M}$ and $\mathbf{C}_{S}$ define a plane inside the RGB color space [3].

By computing chromaticities, the three-dimensional data points are projected onto the plane $r+g+b=1$. This gives us a line in chromaticity space. The two points which define the line are the chromaticities of the measured object color $\left[r_{O}, g_{O}\right]^{T}$ and the chromaticities of the color of the illuminant $\left[r_{E}, g_{E}\right]^{T}$

$$
\left(\begin{array}{l}
r \\
g
\end{array}\right)=s\left(\begin{array}{l}
r_{O} \\
g_{O}
\end{array}\right)+(1-s)\left(\begin{array}{l}
r_{E} \\
g_{E}
\end{array}\right)
$$


for some scaling factor $s$. The data points which belong to a uniformly colored surface will all be distributed along this, so called, dichromatic line. We now assume that the illuminant is uniform over the entire scene. In this case, all dichromatic lines will have one point in common, the color of the illuminant.

\section{$3 \quad$ Natural Illuminants}

If we know the correspondence between data points and surfaces, we can compute the dichromatic line for each surface. The dichromatic line can be found by doing a linear regression on the data. Alternatively we can also compute the covariance matrix and then locate the eigenvector which corresponds to the largest eigenvalue to determine the orientation of the dichromatic line. According to Finlayson and Schaefer [4] the algorithms based on the dichromatic reflection model perform well only under idealized conditions. The estimated illuminant turns out not to be very accurate. If small amounts of noise are present in the data then the computed intersection may be very different from the actual intersection. Finlayson and Schaefer note that the method works well for highly saturated surfaces under laboratory conditions but does not work well for real images. In their work, they assumed the images to be pre-segmented.

They suggest to compute the intersection of the dichromatic lines with the curve of the black-body radiator in order to make the method more robust. Many natural light sources can be approximated by a black-body radiator. The power spectrum $E(\lambda, T)$ of a black-body radiator depends on the temperature $T$. It can be described by the following equation $[5,6]$

$$
E(\lambda, T)=\frac{2 h c^{2}}{\lambda^{5}} \frac{1}{\left(e^{\frac{h c}{k^{T} T \lambda}}-1\right)}
$$

where $T$ is the temperature of the black-body measured in Kelvin, $h=6.626176$. $10^{-34} \mathrm{Js}$ is Planck's constant, $k_{B}=1.3806 \cdot 10^{-23} \frac{\mathrm{J}}{\mathrm{K}}$ is Boltzmann's constant, and $c=2.9979 \cdot 10^{8} \frac{\mathrm{m}}{\mathrm{s}}$ is the speed of light. Many natural light sources such as the flame of a candle, light from a light bulb or sunlight can be approximated by the power spectrum of the black-body radiator. The chromaticities of daylight also follows the curve of the black-body radiator closely [7]. Plotting the chromaticities of the black-body radiator in CIE XYZ color space, one obtains a curve which can be approximated by a quadratic equation.

Using this approximation, we can compute the intersection between the dichromatic line and the curve of the black-body radiator. As a result, one either obtains none, one or two points of intersection. If the dichromatic line does not intersect the curve of the black-body radiator, then one can locate the closest point between the line and the curve of the black-body radiator. If two intersections are found, one can use some heuristics to select one of the two as the correct intersection. Using the constraint that the illuminant can be approximated by the curve of the black-body radiator, in theory it is possible, to determine the color of the illuminant from a single surface. Algorithms based on the gray world assumption $[8,9,10]$ in contrast, require that the scene be sufficiently diverse. 


\section{Estimating the Color of the Illuminant by Segmentation and Filtering}

Risson [11] extended the algorithm of Finlayson and Schaefer by also addressing the segmentation problem. Risson proposed to determine the illuminant by first segmenting the image and then filtering out regions which are not in line with the dichromatic reflection model. As a first step, noise is removed by pre-filtering the image using a Gaussian or median filter. Then the image is segmented. Regions which do not agree with the dichromatic reflection model, such as achromatic regions or regions which belong to the sky, are removed. In order to compute the direction of the dichromatic line reliably, the region has to have a certain size. Risson [11] suggested to remove all regions with a saturation less than $12 \%$. For each remaining region, the dichromatic line is computed.

The dichromatic line can be computed by performing a linear regression on the $\mathrm{x}$ - and $\mathrm{y}$-coordinates in CIE XYZ chromaticity space. We can also compute the covariance matrix for the pixel colors which belong to a single region. Using singular value decomposition, the largest eigenvalue tells us the direction of the dichromatic line. Let $\mathbf{e}_{i}$ be the normalized eigenvector which corresponds to the largest eigenvalue obtained for region $j$. The dichromatic line $\mathcal{L}_{j}$ of region $j$ is then given by

$$
\mathcal{L}_{j}=\left\{\mathbf{a}_{j}+s \mathbf{e}_{j} \mid \text { with } s \in \mathbb{R}\right\}
$$

where $\mathbf{a}_{j}$ is the average chromaticity of the region. In theory, the illuminant is located at the point where all dichromatic lines $\mathcal{L}_{j}$ intersect. In practice, however, the dichromatic lines do not intersect in a single point because of noise in the data. It may also be that some of the computed lines are not caused by pure matte reflections in combination with specular reflections.

It may be possible to develop a classifier to rule out lines which are not in agreement with the dichromatic reflection model. A simpler method is to use the large number of dichromatic lines obtained from the image and to gather statistical evidence for the actual point of intersection. The exact method on how to determine the location of the point of intersection is not specified by Risson [11]. In finding the point of intersection, the curve of the black-body radiator may or may not be used to constrain the set of possible illuminants.

A simple method with no constraints on the color of the illuminant would be to compute the intersection for all possible combinations between two dichromatic lines. This gives us a set of intersections [12]. Let $n$ be the number of dichromatic lines of the image. This gives us $\frac{1}{2} n(n-1)$ points of intersection $\mathbf{p}_{i}=\left[x_{i}, y_{i}\right]$ where $x_{i}$ and $y_{i}$ are the chromaticities in CIE XYZ color space.

$$
\left\{\mathbf{p}_{i} \mid \text { with } i \in\left[1, \ldots, \frac{1}{2} n(n-1)\right\}\right.
$$

From this set we can estimate the actual point of intersection by computing the average of the points of intersection. In this case, the position of the illuminant $\mathbf{p}$ is given by

$$
\mathbf{p}=\frac{2}{n(n-1)} \sum_{i} \mathbf{p}_{i}
$$


We could also compute the median independently for the $\mathrm{x}$ - and $\mathrm{y}$-coordinates. Use of the median operator has the advantage that outliers are removed.

$$
\mathbf{p}=\left[\operatorname{Median}\left\{x_{i}\right\}, \operatorname{Median}\left\{y_{i}\right\}\right]
$$

where Median denotes the computation of the median.

Assuming that the illuminant can be approximated by the curve of the blackbody radiator, we can use this curve to constrain the set of possible illuminants. Thus, for each dichromatic line we would first compute the intersection between each dichromatic line and the curve of the black-body radiator and then further process this data. Since the curve of the black-body radiator is a quadratic function of the single coordinate $\mathrm{x}$ we obtain either none, one or two points of intersection on the dichromatic line. We could take the average or the median value of all computed $\mathrm{x}$ coordinates and then compute the corresponding y coordinate. We could also compute a histogram for all intersection points and then select the bucket with the maximum count. Another possibility would be to determine the corresponding temperature of the black-body radiator for each intersection. Again we could then compute either the average or the median temperature of all intersections. Given the temperature we could compute the corresponding chromaticities in CIE XYZ color space.

Once the illuminant is known, it can be factored out of the image. As a result, we obtain a color image as it would appear under a canonical, e.g. white illuminant. Figure 1 shows the steps of the algorithm for a sample image.

\section{Experimental Results}

In order to evaluate which method of determining the most likely position of the illuminant works best, we have used a standard dataset for color constancy research, the datasets of Barnard et al. [13] ${ }^{1}$. Five different sets were selected from the database. Set 1 contains only objects with minimal specularities, i.e. Lambertian reflectors. Set 2 contains objects with metallic specularities. Set 3 contains objects with non-negligible dielectric specularities. Set 4 contains objects with at least one fluorescent surface. Each object is imaged under up to 11 different illuminants. The objects remained stationary whenever the illuminant was changed. Set 5 can be used to evaluate the performance on object recognition tasks. Objects from set 5 were placed in a random position whenever the illuminant was changed. Sets 1, 2, 3, 4, and 5 contain 22, 14, 9, 6, and 20 different scenes, respectively.

The evaluation of the different methods described in the previous section was done as follows. The color constancy algorithm based on the dichromatic reflection model was applied to each image from the dataset. Then we randomly selected two images from the dataset for each object. This gave us two image sets. The first set of images contains the test images. The second set of images contains the model images. The histogram based object recognition, originally

\footnotetext{
${ }^{1}$ http://www.cs.sfu.ca/ colour/data/index.html
} 


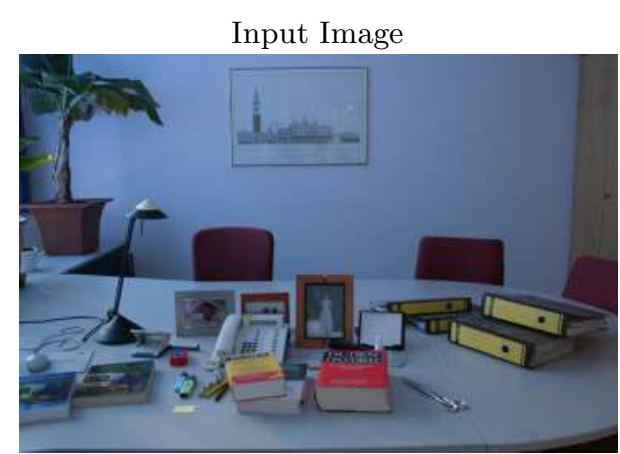

Remaining Regions

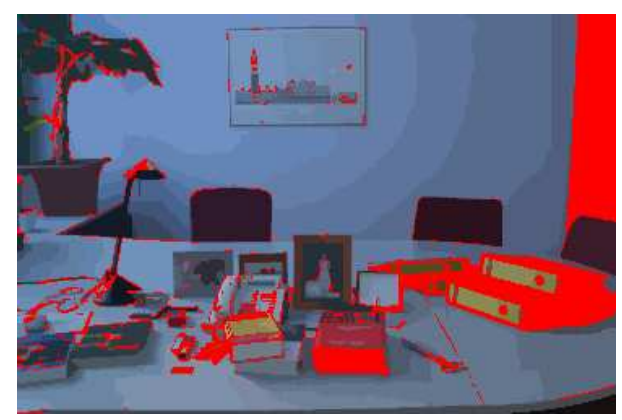

Segmented Image

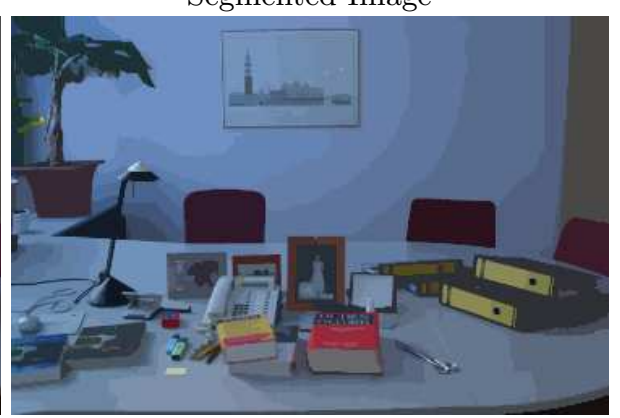

Dichromatic Lines

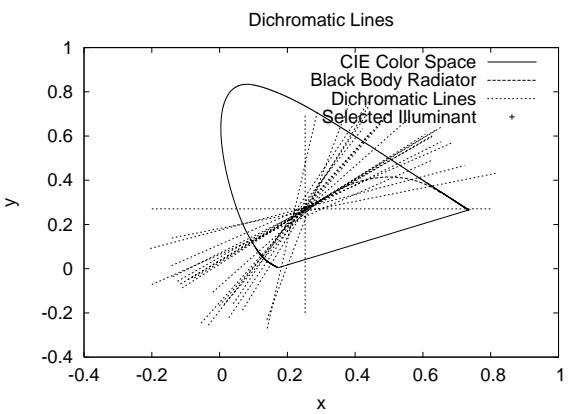

Output Image

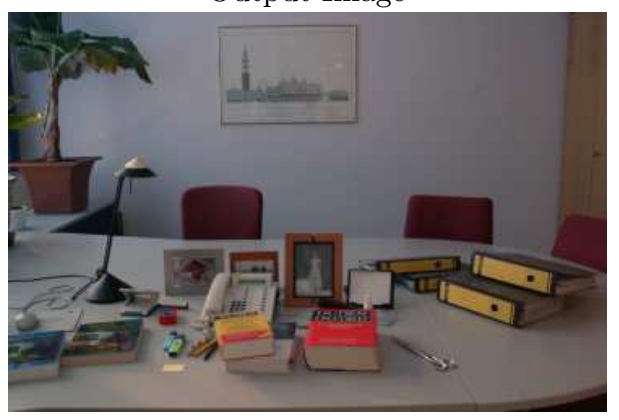

Fig. 1. Steps of Risson's [11] algorithm. The input image is segmented into regions of uniform color. Regions which are not in line with the dichromatic reflection model are removed. Here, small regions and regions with low saturation are removed (marked in red). For each remaining region, the dichromatic line is computed. Only a subset of all lines are shown for clarity. The estimated illuminant is marked by a vertical and horizontal line. The last image shows the output image.

introduced by Swain and Ballard [14], was used to find a match for each test image. Since our goal is to evaluate the different estimation methods we have used only color histograms and did not include any other information, i.e. gradients. 
The $\chi^{2}$ divergence measure $[15,16]$ was used to find the best match. Let $H_{T}$ be the color histogram of the test image and and let $H_{M}$ be the color histogram of the model image. Let $H(\mathbf{c})$ be the probability that the color c occurs in the image then the $\chi^{2}$ divergence measure is computed as

$$
\chi^{2}\left(H_{T}, H_{M}\right)=\sum_{\mathbf{c}} \frac{\left(H_{T}(\mathbf{c})-H_{M}(\mathbf{c})\right)^{2}}{H_{T}(\mathbf{c})+H_{M}(\mathbf{c})} .
$$

This process was repeated 100 times for each data set.

Table 1. Results for image sets 1 through 5 . Histograms were computed in RGB chromaticity space. For each image set random performance is also shown.

Histogram-based Object Recognition, chromaticity space

\begin{tabular}{llllll} 
Estimation Method & 1 & 2 & 3 & 4 & 5 \\
\hline \hline Random Recognition Rate & 0.045 & 0.071 & 0.111 & 0.167 & 0.050 \\
Avg. Intersection & 0.170 & 0.282 & 0.333 & 0.337 & 0.154 \\
Median Intersection & $\mathbf{0 . 5 3 5}$ & $\mathbf{0 . 5 8 9}$ & $\mathbf{0 . 8 3 9}$ & $\mathbf{0 . 7 2 5}$ & $\mathbf{0 . 3 2 8}$ \\
Black-Body Average X & 0.373 & 0.504 & 0.576 & 0.565 & 0.235 \\
Black-Body Median X & 0.359 & 0.461 & 0.580 & 0.580 & 0.243 \\
Black-Body Histogram & 0.339 & 0.429 & 0.608 & 0.533 & 0.251 \\
Black-Body Avg. Temperature & 0.325 & 0.434 & 0.584 & 0.540 & 0.228 \\
Black-Body Med. Temperature & 0.337 & 0.428 & 0.567 & 0.533 & 0.212
\end{tabular}

Table 1 shows the results for each data set and method of estimating the illuminant. The performance based on a random selection of matches is shown at the top of the table. Selecting the median intersection method clearly produced the best results. Since the dichromatic reflection model was developed for objects with specular surfaces it is of no surprise that best results were achieved for set 3. Recognition results for set 5 which presents a more realistic scenario for object recognition are much lower than the results for sets 1 through 4 . Restricting the illuminant to be on the curve of the black-body radiator did not result in better performance compared to the median intersection. If we assume the illuminant to be caused by natural illumination such as sunlight or tungsten light then we automatically rule out a greenish or purple illuminant. The above results show that it makes sense not to make any assumptions on the color of the illuminant.

\section{Conclusion}

Algorithms based on the dichromatic reflection model are especially suited to achieve color constancy provided that specular objects are in the image. In theory, the color of the illuminant is located at the intersection of the dichromatic lines. In practice, however, the dichromatic lines do not intersect in a single point. Thus, one has to decide upon a method to estimate the color of the illuminant from the available data. We evaluated several different methods on how 
to estimate the color of the illuminant using histogram based object recognition. We found that selecting the median of the intersection of the dichromatic lines in CIE chromaticity space gave best results. Also, it is not advantageous to make the assumption that the color of the illuminant can be approximated by the curve of the black-body radiator.

\section{References}

1. Zeki, S.: A Vision of the Brain. Blackwell Science, Oxford (1993)

2. Tominaga, S.: Surface identification using the dichromatic reflection model. IEEE Transactions on Pattern Analysis and Machine Intelligence 13(7) (1991) 658-670

3. D'Zmura, M., Lennie, P.: Mechanisms of color constancy. In Healey, G.E., Shafer, S.A., Wolff, L.B., eds.: Color, Boston, Jones and Bartlett Publishers (1992) 224-234

4. Finlayson, G.D., Schaefer, G.: Solving for colour constancy using a constrained dichromatic reflection model. International Journal of Computer Vision 42(3) (2001) 127-144

5. Haken, H., Wolf, H.C.: Atom- und Quantenphysik: Einführung in die experimentellen und theoretischen Grundlagen. Vierte edn. Springer-Verlag, Berlin, Heidelberg (1990)

6. Jähne, B.: Digitale Bildverarbeitung. Fifth edn. Springer-Verlag, Berlin (2002)

7. International Commission on Illumination: Colorimetry, 2nd edition, corrected reprint. Technical report, International Commission on Illumination (1996)

8. Buchsbaum, G.: A spatial processor model for object colour perception. Journal of the Franklin Institute 310(1) (1980) 337-350

9. Gershon, R., Jepson, A.D., Tsotsos, J.K.: From $[R, G, B]$ to surface reflectance: Computing color constant descriptors in images. In McDermott, J.P., ed.: Proceedings of the Tenth International Joint Conference on Artificial Intelligence, Milan, Italy. Volume 2., Morgan Kaufmann (1987) 755-758

10. Ebner, M.: A parallel algorithm for color constancy. Journal of Parallel and Distributed Computing 64(1) (2004) 79-88

11. Risson, V.J.: Determination of an illuminant of digital color image by segmentation and filtering. United States Patent Application, Pub. No. US 2003/0095704 A1 (2003)

12. Herrmann, C.: Schätzung der Farbe einer Lichtquelle anhand eines digitalen Bildes durch Segmentierung und Filterung. Projektpraktikum, Universität Würzburg, Institut für Informatik, Lehrstuhl für Informatik II (2004)

13. Barnard, K., Martin, L., Funt, B., Coath, A.: A data set for color research. Color Research and Application 27(3) (2002) 147-151

14. Swain, M.J., Ballard, D.H.: Color indexing. International Journal of Computer Vision 7 (1991) 11-32

15. Schiele, B., Crowley, J.L.: Object recognition using multidimensional receptive field histograms. In Buxton, B., Cipolla, R., eds.: Fourth European Conference On Computer Vision, Cambridge, UK, April 14-18, Berlin, Springer-Verlag (1996) 610-619

16. Schiele, B., Crowley, J.L.: Recognition without correspondence using multidimensional receptive field histograms. International Journal of Computer Vision 36(1) (2000) $31-52$ 